\title{
Inverse Problem Related to Boundary Shape Identification for a Hyperbolic Differential Equation
}

\author{
Fagueye Ndiaye (iD) and Idrissa Ly \\ Laboratory of Mathematics of Decision and Numerical Analysis, Cheikh Anta Diop University, BP 5036 Dakar-Fann, Senegal \\ Correspondence should be addressed to Fagueye Ndiaye; fagueye.ndiaye@ucad.edu.sn
}

Received 12 August 2021; Accepted 11 September 2021; Published 18 October 2021

Academic Editor: Remi Léandre

Copyright (C) 2021 Fagueye Ndiaye and Idrissa Ly. This is an open access article distributed under the Creative Commons Attribution License, which permits unrestricted use, distribution, and reproduction in any medium, provided the original work is properly cited.

In this paper, we are interested in the inverse problem of the determination of the unknown part $\partial \Omega, \Gamma_{0}$ of the boundary of a uniformly Lipschitzian domain $\Omega$ included in $\mathbb{R}^{N}$ from the measurement of the normal derivative $\partial_{n} v$ on suitable part $\Gamma_{0}$ of its boundary, where $v$ is the solution of the wave equation $\partial_{t t} v(x, t)-\Delta v(x, t)+p(x) v(x)=0$ in $\Omega \times(0, T)$ and given Dirichlet boundary data. We use shape optimization tools to retrieve the boundary part $\Gamma$ of $\partial \Omega$. From necessary conditions, we estimate a Lagrange multiplier $k(\Omega)$ which appears by derivation with respect to the domain. By maximum principle theory for hyperbolic equations and under geometrical assumptions, we prove a uniqueness result of our inverse problem. The Lipschitz stability is established by increasing of the energy of the system. Some numerical simulations are made to illustrate the optimal shape.

\section{Introduction and Main Result}

The inverse problem in this paper means the problem of reconstructing object from observation data. We restrict ourselves to the case when the observation data are given as a boundary of the Cauchy data of a solution of a wave equation and the unknown object is a boundary. Let $N \in \mathbb{N}, T>0$ and let $\Omega \subset \mathbb{R}^{N}$ be a bounded domain with smooth boundary $\partial \Omega$. Moreover, let us consider a partition of this boundary $\partial \Omega=\Gamma_{0} \cup \Gamma, \Gamma_{0} \cap \Gamma=\varnothing$, where $\Gamma_{0}$ is the accessible regular part, for example, $\mathscr{C}^{2}$, and it satisfies the interior sphere condition (see [1]) and $\Gamma=\partial \Omega / \Gamma_{0}$ is the unknown part of boundary. Throughout this paper, let us take the functional $v=v(x, t)$ with $x \in \Omega, t \in(0, T)$. We use the following notations:

$$
\partial_{n} v=\frac{\partial v}{\partial n}, \partial_{t t} v(x, t)=\frac{\partial^{2} v}{\partial t^{2}}
$$

We consider the following wave equation:

$$
\left\{\begin{array}{l}
\frac{\partial^{2} v}{\partial t^{2}}+p(x) v-\Delta v=0 \text { in } \Omega \times(0, T), \\
v(x, t)=0 \text { on } \partial \Omega \times(0, T), \\
v(x, 0)=v_{0}(x) \text { in } \Omega \\
\frac{\partial}{\partial t} v(x, 0)=v_{1}(x) \text { in } \Omega .
\end{array}\right.
$$

First of all, assume that $p \in L^{\infty}(\Omega), \quad v_{0} \in H^{1}(\Omega)$, and $v_{1} \in L^{2}(\Omega)$ are given and verified the compatibility condition $v_{0}(x)=0$ for all $x \in \partial \Omega$. The Cauchy problem (2) is known to be well posed and one can also prove the solution $v \in C\left([0, T] ; H_{0}^{1}(\Omega)\right) \cap C^{1}\left([0, T] ; L^{2}(\Omega)\right)$; this result can be found in [2].

Our inverse problem consists of determining $\Gamma=\partial \Omega, \Gamma_{0}$, the unknown part of boundary from Cauchy data $\left(\left.v_{0}\right|_{\partial \Omega}, h\right)$ of a weak solution $v$ of the following problem (3) with a given potential $p(x)$. 


$$
\left\{\begin{array}{l}
\frac{\partial^{2} v}{\partial t^{2}}+p v-\Delta v=0 \text { in } \Omega \times(0, T) \\
v(x, t)=0 \text { on } \partial \Omega \times(0, T) \\
v(x, 0)=v_{0}(x) \text { in } \Omega \\
\frac{\partial}{\partial t} v(x, 0)=v_{1}(x) \text { in } \Omega \\
\frac{\partial v}{\partial n}(x, t)=h(x) \text { on } \Gamma_{0} \times(0, T)
\end{array}\right.
$$

where $h$ is a given function, and the corresponding Neumann data measured on $\Gamma_{0}$ and $n$ is outer normal vector unit. In this case, $\Omega$ and $v$ are unknowns and we assume that the normal derivatives of function $v$ can be measured by $h$.

In Section 1, we present the inverse problem which consists of finding a formula reconstructing the part of boundary $\Gamma$ from the Cauchy data. The remainder of the paper is organized as follows.

In Section 2, we establish the shape optimization problem and prove the existence results. In Section 3, we study the derivation with respect to the domain and we prove the necessary conditions of optimality, that is, the existence of a Lagrange multiplier. Section 4 is devoted to auxiliary lemmas based on maximum principle theory for hyperbolic equations; see [3]. In Section 5, we give by a monotonicity result and under geometrical assumptions a uniqueness result of our inverse problem.

The questions for the wave equation have all already received positive answers since the uniqueness result for the linear inverse problem has been proved by Klibanov (see [4]) and Lipschitz stability results (for both linear and nonlinear inverse problems) of Yamamoto (see [5]). Many results, to which we can refer concerning the wave equation, are related to the same type of inverse problem of determining a potential $p(x)$. Some of them can be found in [6], for example. These references are all based upon local Carleman estimates for the wave operator (see [5]) or global Carleman estimates for Schrödinger equation (see [7]) to prove uniqueness and stability estimate solution. Nevertheless, in our approach, for a given potential, the reconstruction of $\Gamma$ from the Cauchy data is one of our aims and the estimation of the Lagrange multiplier which appears by derivation with respect to the domain of the energy of system in an admissible set of domains is another interesting one.

In [8], Isakov and Friedman studied the inverse spectral problems. This domain problem was formulated already by Sir A. Shuster who in 1882 introduced spectroscopy as a way to find a shape of a bell by means of the sounds which it is capable of sending out. More rigorously, it has been posed by Bochner in the 1950s and then in the well-known lecture of Kac (see [9]) “Can one hear the shape of a drum?" in 1966. $\mathrm{He}$ also studied inverse problem of gravimetry, inverse conductivity problem, tomography, and the inverse seismic problem and indicated their applications.
In [10], using conformal mapping technique, Kress studied mathematical modelling of electrostatic or thermal imaging methods in nondestructive testing and evaluation. In these applications, an unknown inclusion within a conducting host medium with constant conductivity is assessed from overdetermined Cauchy data on the accessible exterior boundary of the medium.

\section{Study of the Shape Optimization Problem}

2.1. Auxiliary Results. We describe some fundamental properties which will be useful in the following. We consider a fixed and bounded domain $D$ in $\mathbb{R}^{N}$ which contains all open subsets we used.

Definition 1. Let $K_{1}$ and $K_{2}$ be two compact subsets of $D$. Let

$$
\begin{aligned}
& d\left(x, K_{1}\right)=\min _{y \in K_{1}} d(x, y), \\
& d\left(x, K_{2}\right)=\min _{y \in K_{2}} d(x, y) .
\end{aligned}
$$

Note that

$$
\begin{aligned}
& \rho\left(K_{1}, K_{2}\right)=\max _{x \in K_{2}} d\left(x, K_{1}\right), \\
& \rho\left(K_{2}, K_{1}\right)=\min _{x \in K_{1}} d\left(x, K_{2}\right) .
\end{aligned}
$$

Let

$$
d_{H}\left(K_{1}, K_{2}\right)=\max \left[\rho\left(K_{1}, K_{2}\right), \rho\left(K_{2}, K_{1}\right)\right],
$$

and we call Hausdorff distance of $K_{1}$ and $K_{2}$, the following positive number, denoted by $d_{H}\left(K_{1}, K_{2}\right)$.

Let $\left(\Omega_{n}\right)$ be a sequence of open subsets of $D$ and let $\Omega$ be an open subset of $D$. We say that the sequence $\left(\Omega_{n}\right)$ converges on $\Omega$ in the Hausdorff sense and we denote it by $\Omega_{n} \stackrel{H}{\longrightarrow} \Omega$ if $\lim d_{H}\left(\bar{D} \backslash \Omega_{n}, \bar{D} \backslash \Omega\right)=0$.

Let $\left(\Omega_{n}\right)$ be a sequence of open sets of $\mathbb{R}^{N}$ and let $\Omega$ be an open set of $\mathbb{R}^{N}$. We say that the sequence $\left(\Omega_{n}\right)$ converges on $\Omega$ in the sense of $L^{p}, 1 \leq p<\infty$ if $\chi_{\Omega_{n}}$ converges on $\chi_{\Omega}$ in $L_{\text {loc }}^{p}\left(\mathbb{R}^{N}\right), \chi_{\Omega}$ being the characteristic functions of $\Omega$.

Remark 1. Let $\left\{K_{n}\right\}$ be a sequence of compact sets included in a fixed and bounded set $D$ of $\operatorname{IR}^{N}$; then there are a compact set $K$ and $n_{k}$ such that $K_{n_{k}}$ converges on $K$ in the sense of Hausdorff.

We have the following lemmas.

Lemma 1. Let $\left(\Omega_{n}\right)_{n \in I N}$ be a sequence of open set in $I R^{N}$ having the $\epsilon$-cône property with $\bar{\Omega}_{n} \subset F \subset D$, with $F$ being a compact set. Then there exists an open set $\Omega$ including $F$ which satisfies the $\epsilon / 2$-cône property and a subsequence $\Omega_{n k}$ such that

$\chi_{\Omega_{n_{k}}} \stackrel{L^{1}}{\longrightarrow} \chi_{\Omega}, \Omega_{n_{k}} \stackrel{H}{\longrightarrow} \Omega, \partial \Omega_{n_{k}} \stackrel{H}{\longrightarrow} \partial \Omega, \bar{\Omega}_{n_{k}} \stackrel{H}{\longrightarrow} \bar{\Omega}$.

For detailed proof, see [11].

Lemma 2. Let $\left(\Omega_{n}\right)_{n \in \mathbb{N}}$ be a sequence of open sets having the $\epsilon$-cône property and converging to $\Omega$ in the sense of Hausdorff. 
If $v_{\Omega_{n}}$ is the solution of problem 2 in $\Omega_{n}$ for all $n \in \mathbb{N}$ and $v_{\Omega}$ is the solution of this problem in $\Omega$, then $v_{\Omega_{n}}$ converges to $v=v_{\Omega}$.

For detailed proof, see [11].

Lemma 3. Let $\Omega$ be a Lipschitz domain with $v$ in $W^{1, p}\left(\mathbb{R}^{\mathbb{N}}\right)$; if $v=0$ a.e in $\Omega^{c}$, then $v$ in $W_{0}^{1, p}(\Omega)$.

For detailed proof, see [11].

For all $v(x, t)$ solution of

$$
\left\{\begin{array}{l}
\frac{\partial^{2} v}{\partial t^{2}}+p v-\Delta v=0 \text { in } \Omega \times(0, T), \\
v(x, t)=0 \text { on } \partial \Omega \times(0, T), \\
v(x, 0)=v_{0}(x) \text { in } \Omega, \\
\frac{\partial}{\partial t} v(x, 0)=v_{1}(x) \text { in } \Omega,
\end{array}\right.
$$

at time $t$, the energy of $v$ is defined by

$$
E(v, t)=\frac{1}{2} \int_{\Omega}\left|\frac{\partial v}{\partial t}(x, t)\right|^{2} \mathrm{~d} x+\frac{1}{2} \int_{\Omega}|\nabla v(x, t)|^{2} \mathrm{~d} x,
$$

and it verifies

$$
E(v, 0)-E(v, t)=\frac{1}{2} \int_{\Omega} p|v(x, t)|^{2} \mathrm{~d} x-\frac{1}{2} \int_{\Omega} p\left|v_{0}(x)\right|^{2} \mathrm{~d} x .
$$

Let $J$ be the functional defined by

$$
J\left(v_{w}, w\right)=\frac{1}{2} \int_{w}\left|\frac{\partial v_{w}}{\partial t}(x, t)\right|^{2} \mathrm{~d} x+\frac{1}{2} \int_{w}\left|\nabla v_{w}(x, t)\right|^{2} \mathrm{~d} x+\frac{1}{2} \int_{w} p\left|v_{w}(x, t)\right|^{2} \mathrm{~d} x, \quad t \in[0, T]
$$

where $v_{w}(x, t)$ is the solution:

$$
\left\{\begin{array}{l}
\frac{\partial^{2} v}{\partial t^{2}}+p v-\Delta v=0 \text { in } w \times(0, T), \\
v(x, t)=0 \text { on } \partial w \times(0, T), \\
v(x, 0)=v_{0}(x) \text { in } w, \\
\frac{\partial}{\partial t} v(x, 0)=v_{1}(x) \text { in } w .
\end{array}\right.
$$

We study the existence of the result of the following optimization problem: $\inf \{J(w), w \in \mathcal{O}\}$, where the class of admissible domains is defined by

$$
\mathcal{O}=\left\{\omega \subset \mathbb{R}^{N}, \omega \text { domain uniformly Lipschizian included in } D, \int_{\omega} \mathrm{d} x=V_{o}\right\},
$$

where $D$ is a bounded domain of $\mathbb{R}^{N}$ containing all $\Omega$ and $V_{0}$ is positive real.

Remark 2. Remark that $\omega$ being uniformly Lipschitz means $\omega$ satisfies the $\epsilon$-cone property; for details, see [11].

2.2. Existence of Solution of Shape Optimization Problem. We study the existence result of the following shape optimization problem.

Proposition 1. "Find $\omega$ belonging to $\mathcal{O}$ such that $J(\omega)=$ $\min \{J(w), w \in \mathcal{O}\}$ has a solution."
Proof 1. For the proof, we take the following:

$$
\begin{aligned}
& J(w):=E\left(v_{w}, t\right)+1 / 2 \int_{w} p\left|v_{w}(x, t)\right|^{2} \mathrm{~d} x \\
& J(w)>0, \text { which implies } \inf \{J(w), w \in \mathcal{O}\}>-\infty
\end{aligned}
$$

Let $\alpha=\inf \{J(w), w \in \mathcal{O}\}$. Therefore, there is a minimizing sequence $\left(\Omega_{n}\right)_{n \in \mathbb{N}} \in \mathcal{O}$ such that $J\left(\Omega_{n}\right)$ converges to $\alpha$.

The fact that sequence $\left(\Omega_{n}\right)_{n \in \mathbb{N}} \in \mathcal{O}$ is bounded ensures the existence of a subsequence $\left(\Omega_{n_{k}}\right)_{n_{k} \in \mathbb{N}} \in \mathcal{O}$ and a domain $\Omega \in \mathcal{O}$ such that $\left(\Omega_{n_{k}}\right)_{n_{k}}$ converges to $\Omega$ in the sense of Hausdorff according to Lemma 1. 
Therefore, we consider $\quad\left(v_{\Omega_{n}}, t\right)=\widetilde{v}_{n} \quad$ and
$\tilde{p}= \begin{cases}p, \quad x \in \Omega_{n}, \\ 0, \quad x \in \mathbb{R}^{n} \backslash \Omega_{n},\end{cases}$
Sequence $\quad\left(\widetilde{v}_{n}\right)_{n \in N} \quad$ is $\quad$ bounded in $C\left([0, T] ; H^{1}(D)\right) \cap C^{1}\left([0, T] ; L^{2}(D)\right)$. If not, $J\left(\Omega_{n}\right)$ converges to $+\infty$, which is a contradiction.
Space $H^{1}(D) \cap L^{2}(D)$ is reflexive; then there is a subsequence $\left(\widetilde{v}_{n_{k}}\right)_{k \in N}$ et $v^{*}$ such that $v_{n_{k}}$ converges weakly to $v^{*}$ in $C\left([0, T] ; H_{0}^{1}(D)\right) \cap C^{1}\left([0, T] ; L^{2}(D)\right)$ and always according to Lemma 3.

$$
\int_{\Omega}\left(\left|\frac{\partial v^{*}}{\partial t}(x, t)\right|^{2} \mathrm{~d} x+p\left|v^{*}(x, t)\right|^{2} \mathrm{~d} x+\left|\nabla v^{*}\right|^{2}\right)<\liminf \int_{\Omega_{n_{k}}}\left(\left|\frac{\partial \widetilde{v}_{n_{k}}}{\partial t}(x, t)\right|^{2} \mathrm{~d} x+\widetilde{p}\left|\widetilde{v}_{n_{k}}(x, t)\right|^{2} \mathrm{~d} x+\left|\nabla \widetilde{v}_{n_{k}}\right|^{2}\right)
$$

$\forall t \in[0, T]$.

Thus, we obtain $J(\Omega) \leq \liminf J\left(\Omega_{n_{k}}\right)$. Therefore, $J(\Omega) \leq \inf \{J(w), w \in \mathcal{O}\}$; then

$$
J(\Omega)=\min \{J(w), w \in \mathcal{O}\} .
$$

Remark 3. It is easy to verify that $v^{*}$ equals $v_{\Omega}$ according to Lemma 1 and satisfies problem (12). On the other hand, we have a regularity of $v_{\Omega}$ solution to problem $(12)$ (see $\left.[12,13]\right)$.

\section{Derivation with respect to the Domain}

These results would allow us to assume regularity $C^{2}$ on $\Omega$ solution of the shape optimization problem to proceed with the derivation with respect to the domain and to show the result of monotony.

Let $E: \mathcal{O} \longrightarrow X$, with $\mathcal{O}$ being the set of domains having the $\epsilon$-cône property and $X$ being a normal vector space.

Let us consider $\theta \longrightarrow \xi(\theta)=E(I+\theta)(\Omega)$, where $\theta$ varies around 0 in a normalized vector space $\Theta$ of applications from $\mathbb{R}^{N}$ to $\mathbb{R}^{N}$. We can introduce the differentiability in the classic sense of Frechet for the application $\theta \in \Theta \longrightarrow \xi(\theta) \in X$. This is efficient in proving the regularity properties of the shape functional, in using the derivation calculations, and in clearly identifying the derivatives structures so-called "of shape".

We will use a numerical variable to be comfortable in the calculations.

Let us choose function $\phi: y \mapsto I+y \theta$ with $\theta$ being a regular vector field from $\mathbb{R}^{N}$ to $\mathbb{R}^{N} ; y \longrightarrow \phi(y) \in \Theta$. We analyse the derivative of $\phi(y)$ and the expression of $y \in\left[0, Y\left[\longrightarrow E\left(\Omega_{y}\right)\right.\right.$, where $\Omega_{y}=\phi(y)(\Omega)$.

Let us consider $v_{y}=v_{\Omega_{y}}$. The question is, how can we derive the function $y \mapsto v_{y} \in H^{1}\left(\Omega_{y}\right)$ where $\Omega_{y}$ is a variable domain?

(1) We know that $v_{y}$ is extended by 0 because $v_{y} \in H_{0}^{1}\left(\Omega_{y}\right)$

(2) We know that function $u_{y}=v_{y}{ }^{\circ} \phi(y)$ is always defined on the fixed domain $\Omega$; it belongs to space $H^{1}(\Omega)$

To derive function $v_{y}$, it suffices to "transport" it by $\phi_{y}$, because $y \mapsto u_{y}$ has more regularity than $y \mapsto v_{y}$. Therefore, it is more strategic to study this problem.

We fix $\Omega \subset \mathbb{R}^{N}$ as measurable. It is easy to verify that $\Omega_{y}$ is measurable and that if $\Omega$ is open, then $\Omega_{y}$ is also measurable.
3.1. Notations. Let $W^{1, \infty}\left(\mathbb{R}^{N}, \mathbb{R}^{N}\right)$ denote the bounded space, and Lipschitzian applications from $\mathbb{R}^{N}$ in itself provide with the norm

$$
\forall \theta \in W^{1, \infty},\|\theta\|_{1, \infty}=\sup _{y, \widehat{y} \in \mathbb{R}^{N}, y \neq \hat{y}}\left\{|\theta(y)|+\frac{|\theta(y)-\theta(\widehat{y})|}{|y-\hat{y}|}\right\},
$$

where $\mathbb{R}^{N}$ is provided with the Euclidean norm $\|$. Let $I$ denote the identity of $\mathbb{R}^{N}$.

We recall that this space is identified with the subspace of $L^{\infty}\left(\mathbb{R}^{N}\right)$, whose partial derivatives in the sense of distributions are functions of $L^{\infty}\left(\mathbb{R}^{N}\right)$. In addition, the functions of $W^{1, \infty}$ are a.e. differentiable, and we have

$$
\forall \theta \in W^{1, \infty}, \quad\|\theta\|_{1, \infty}=\|\theta\|_{\infty}+\underset{y \in \mathbb{R}^{N}}{\operatorname{esssup}}\left\|D_{y} \theta(y)\right\|,
$$

where the norms of differential are understood as linear operators of $\mathbb{R}^{N}$. However, the consideration of $W^{1, \infty}$ is interesting for deformations of the Lipschitzian domain.

If $\|\theta\|_{1, \infty}<1$, by the theorem of fixed point, $1+\theta$ is inversible such that $(1+\theta)^{-1} \in W^{1, \infty}$ and we have (see [11])

$$
\left\{\begin{array}{l}
\left\|(I+\theta)^{-1}-I\right\|_{1, \infty} \leq\|\theta\|_{1, \infty}\left(I-\|\theta\|_{1, \infty}\right)^{-1} \\
\left\|(I+\theta)^{-1}-I+\theta\right\|_{\infty} \leq\|\theta\|_{1, \infty}\left\|I-(I+\theta)^{-1}\right\|_{\infty}
\end{array}\right.
$$

Thus,

$$
\begin{aligned}
& \theta \in W^{1, \infty} \longrightarrow(I+\theta)^{-1} \in W^{1, \infty} \text { is continuous in } 0 \\
& \theta \in W^{1, \infty} \longrightarrow(I+\theta)^{-1} \in L^{\infty} \text { is differentiable in } 0, \\
& \text { and its differentiability is the opposite of identity }
\end{aligned}
$$

Let us consider $\phi: y \in\left[0 ; Y\left[\longrightarrow W^{1, \infty}\left(\mathbb{R}^{N}\right)\right.\right.$ derivable in 0 with

$$
\phi(0)=I, \phi^{\prime}(0)=V .
$$

Because $\phi(y)$ is close to the identity in $W^{1, \infty}\left(\mathbb{R}^{N}\right)$ for $y$ close to 0 , it is inversible and even if it decreases $Y$ according to 7 .

$$
\left\{\begin{array}{l}
t \in\left[0 ; Y\left[\longrightarrow \phi(y)^{-1} \in W^{1, \infty} \text { is continuous in } 0,\right.\right. \\
t \in\left[0 ; Y\left[\longrightarrow \phi(y)^{-1} \in L^{\infty} \text { is derivable in } 0, \text { with derivative }-V .\right.\right.
\end{array}\right.
$$

We write independently $\phi(y)(x)$ or $\phi(y, x)$ (same for all other functions). Let $J(y, x)=\operatorname{det}\left(D_{x} \phi(y)(x)\right)$ be the 
Jacobian of $\phi(y)$ in $x$ (which is, therefore, a.e. defined in $x \in \mathbb{R}^{N}$ ).

For all $y \in\left[0 ; Y\left[\right.\right.$, we take $f(y,.) \in L^{1}\left(\Omega_{y}\right)$ and consi$\operatorname{der} y \in\left[0 ; Y\left[\longrightarrow I(y)=\int_{\Omega_{y}} f(y, x) \mathrm{d} x=\int_{\Omega} f(y, \phi(y, x))\right.\right.$ $J(y, x) \mathrm{d} x$.

Subsequently, we note $\int_{\Omega_{y}} f(y)=\int_{\Omega} f(y, \phi(y)) J(y)$.
3.2. Derivation Formula. To calculate derivation, we use the following theorem.

Theorem 3.1. Let us consider $\phi$ verifying (19). We suppose that

$$
\begin{aligned}
& y \in\left[0 ; Y\left[\longrightarrow f(y) \in L^{1}\left(\mathbb{R}^{N}\right) \text { is derivable in } 0, \text { of derivative } f^{\prime}(0),\right.\right. \\
& f(0) \in W^{1,1}\left(\mathbb{R}^{N}\right) .
\end{aligned}
$$
have

Then, $t \longrightarrow I(y)=\int_{\Omega_{y}} f(y)$ is derivable in 0 , and we

$$
I^{\prime}(0)=\int_{\Omega} f^{\prime}(0)+\operatorname{div}[f(0) V]
$$

If, in addition, $\Omega$ is a Lipschitzian domain, then

$$
I^{\prime}(0)=\int_{\Omega} f^{\prime}(0)+\int_{\partial \Omega} f(0) n . V .
$$

For detailed proof, see [11].

3.3. Optimality Conditions of the Problem. Again, $y \mapsto \phi(y)$ verifying (19), $\Omega \subset \mathbb{R}^{N}$ is open bounded Lipschitzian domain, and $\Omega_{y}=\phi(y)(\Omega)$. If $y$ is close to $0, v_{y}$ is a solution of the problem defined by its variational formulation (3). As in (11), we are interested in the following functional:

$$
J\left(v_{y}, \Omega_{y}\right)=\frac{1}{2} \int_{\Omega_{y}}\left|\frac{\partial v_{y}}{\partial t}(x, t)\right|^{2} \mathrm{~d} x+\frac{1}{2} \int_{\Omega_{y}}\left|\nabla v_{y}(x, t)\right|^{2} \mathrm{~d} x+\frac{1}{2} \int_{\Omega} p|v(x, t)|^{2} \mathrm{~d} x .
$$

We set

(i) $\Omega_{y}=(I d+y \theta) \Omega$ with $\theta \in W^{1, \infty}\left(\mathbb{R}^{d}, \mathbb{R}^{d}\right)$, a vector field with compact support and $y$ sufficiently small such that $I d+y \theta$ defines a diffeomorphism

(ii) $I(y)=J\left(\Omega_{y}\right)=J\left(v_{\Omega_{y}}, \Omega_{y}\right)$
As regards derivative of $J$, for a choice of $\theta$, as mentioned above, we deform only the boundary part $\partial \Omega \backslash \Gamma_{0}$ that we will denote by $\Gamma$.

To calculate the derivative $y \mapsto I(y)$, it is useful to derive $y \mapsto v_{y}$ in the appropriate direction $v_{y} \in H_{0}^{1}\left(\Omega_{y}\right)$.

For the functional derivative, we have

We look for the derivative of $J$ with respect to domain $\Omega$ in direction $\theta$, that is, $I^{\prime}(0)$.

$$
\begin{aligned}
I^{\prime}(0)= & <\mathrm{d} J\left(v_{\Omega}, \Omega\right), \theta>=\int_{\Omega} \frac{\partial v}{\partial t} \cdot \frac{\partial v^{\prime}}{\partial t} \mathrm{~d} x+\int_{\Omega} \nabla v \cdot \nabla v^{\prime} \mathrm{d} x+\int_{\Omega} p v \cdot v^{\prime} \mathrm{d} x, \\
& +\frac{1}{2} \int_{\partial \Omega}\left|\frac{\partial v}{\partial t}(x, t)\right|^{2}(\theta . n) \mathrm{d} \sigma+\frac{1}{2} \int_{\partial \Omega}|\nabla v(x, t)|^{2}(\theta . n) \mathrm{d} \sigma+\frac{1}{2} \int_{\partial \Omega} p|v(x, t)|^{2}(\theta . n) \mathrm{d} \sigma .
\end{aligned}
$$

This gives, according to Hadamard and the boundary conditions,

$$
\begin{aligned}
I^{\prime}(0)= & <\mathrm{d} J\left(v_{\Omega}, \Omega\right), \theta>=-\frac{1}{2} \int_{\partial \Omega}\left(\frac{\partial v}{\partial n}\right)^{2}(\theta . n) \mathrm{d} \sigma+\frac{1}{2} \int_{\Omega} \frac{\partial^{2}}{\partial t^{2}} v v^{\prime} \mathrm{d} x+\int_{\Omega} v^{\prime} \frac{\partial^{2} v}{\partial t^{2}} \mathrm{~d} x+\int_{\Omega} \nabla v \cdot \nabla v^{\prime} \mathrm{d} x+\int_{\Omega} p v \cdot v^{\prime} \mathrm{d} x, \\
& +\frac{1}{2} \int_{\partial \Omega}\left|\frac{\partial v}{\partial t}(x, t)\right|^{2}(\theta . n) \mathrm{d} x+\frac{1}{2} \int_{\partial \Omega}|\nabla v(x, t)|^{2}(\theta . n) \mathrm{d} x+\frac{1}{2} \int_{\partial \Omega} p|v(x, t)|^{2}(\theta . n) \mathrm{d} x .
\end{aligned}
$$


Therefore,

$$
I^{\prime}(0)=<\mathrm{d} J\left(v_{\Omega}, \Omega\right), \theta>=-\int_{\Gamma}|\nabla v|^{2}(\theta . n) .
$$

We suppose that it is possible to estimate the normal derivative of $v_{\Omega}(x, t)$ on $\Gamma_{0}$; that is, there exists $h: \mathbb{R}^{N} \longrightarrow \mathbb{R}_{-}^{*}$ such that $x \mapsto \partial v(x) / \partial n=h(x)$ on $\Gamma_{0}$, where $n$ is the exterior normal unit vector defined on $\Gamma_{0}$. We have the following necessary conditions of optimality.

Proposition 2. If $\Omega$ is the solution of the shape optimization problem $\min \{J(w), w \in \mathcal{O}\}$, there exists a Lagrange multiplier $k(\Omega)<0$ not depending on $t$ such that $|\nabla v|=-\partial v_{\Omega} / \partial n=(-k(\Omega))^{1 / 2}$ on $\Gamma$.

Proof 2. As $J(\Omega)=\inf \{J(\omega), \omega \in \mathcal{O}\}$, using the derivative with respect to domain, in the direction of vector field, we show that there exists $k(\Omega)$ Lagrange multiplier such that

$$
\mathrm{d} J(\Omega, \theta)=k(\Omega) \mathrm{d} J_{1}(\Omega, \theta),
$$

where $J_{1}(\Omega)=\int_{\Omega} d x-V_{0}$. Note that we perturb $\Omega$ only on $\partial \Omega \backslash \Gamma_{0}=\Gamma, \Gamma_{0}$ is fixed.

$$
\begin{aligned}
\mathrm{d} J(\Omega, \theta) & =k(\Omega) \mathrm{d} J_{1}(\Omega, \theta)=k(\Omega) \int_{\Gamma} \theta . n \\
& =-\int_{\Gamma}|\nabla v|^{2}(\theta . n) \Leftrightarrow\left|\nabla v_{\Omega}\right|^{2}=-k(\Omega) \text { on } \Gamma .
\end{aligned}
$$

This gives us the following relationship according to (27):

$$
k(\Omega)=-|\nabla v|^{2} .
$$

For more details on the expression in (29), see [14].

Let us take $v_{\Omega}=v$. To estimate $k(\Omega)$, it suffices to recognize $\Omega$ and if we suppose that $\partial \Omega \backslash \Gamma_{0}=\Gamma$ is of class $C^{2}$, since $v=0$ on $\Gamma$, then we have

$$
|\nabla v|=-\frac{\partial v}{\partial n}, \text { on } \Gamma
$$

where $n$ is the outer normal vector. Note that $|\nabla v|=(-k(\Omega))^{1 / 2}$ is an optimality condition and if we situate $\Gamma$, we will be able to estimate $|\nabla v|$ on $\Gamma$. Therefore, we deduce an approximation of Lagrange multiplier $k(\Omega)$.

\section{Auxiliary Lemmas}

In this section, we sum up some fundamental lemmas for the algorithm which we will present in the next sections. These lemmas are based only on maximum principle theory in wave equation in high dimension. We assume also that $u_{i} \in C^{2}(\Omega) \cap C\left(\bar{\Omega} \backslash \Gamma_{i}\right), \quad i=1,2$.

In sequel, we need some hypothesis for the operator and the initial value problem in order to apply maximum principle for hyperbolic problem to obtain additional information about functions which satisfy

$$
(\mathscr{H})\left\{\begin{array}{l}
\frac{\partial^{2} v}{\partial t^{2}}+p v-\Delta v \geq 0 \text { in } \Omega \times(0, T), \\
v(x, t)=0 \text { on } \partial \Omega \times(0, T)=\Gamma_{0} \times(0, T), \\
v(x, 0)=v_{0}(x) \leq 0 \text { in } \Omega, \\
\frac{\partial}{\partial t} v(x, 0)=v_{1}(x) \leq 0 \text { in } \Omega .
\end{array}\right.
$$

If the hypothesis $(\mathscr{H})$ holds, then the solution $v$ satisfies $v \leq 0$, for all $t>0$; see [3], page 234 .

Lemma 4. Let $\Omega, \Omega^{\prime} \subset I R^{N}$ be two open sets such that $\Omega^{\prime} \subset \Omega$ and $\Gamma_{0} \subset \partial \Omega \cap \partial \Omega^{\prime}$. We consider $(\mathscr{H})$ and

$$
\left\{\begin{array}{l}
\frac{\partial^{2} v}{\partial t^{2}}+p v-\Delta v=0 \text { in } \Omega \times(0, T), \\
v(x, t)=0 \text { on } \partial \Omega \times(0, T)=\Gamma_{0} \times(0, T), \\
v(x, 0)=v_{0}(x) \operatorname{in} \Omega, \\
\frac{\partial}{\partial t} v(x, 0)=v_{1}(x) \text { in } \Omega,
\end{array}\right.
$$

$$
\left\{\begin{array}{l}
\frac{\partial^{2} v^{\prime}}{\partial t^{2}}+p v^{\prime}-\Delta v^{\prime}=0 \text { in } \Omega^{\prime} \times(0, T), \\
v^{\prime}(x, t)=0 \text { on } \partial \Omega^{\prime} \times(0, T)=\Gamma_{0} \times(0, T), \\
v^{\prime}(x, 0)=v_{0}^{\prime}(x) \text { in } \Omega^{\prime}, \\
\frac{\partial}{\partial t} v^{\prime}(x, 0)=v_{1}^{\prime}(x) \text { in } \Omega^{\prime}
\end{array}\right.
$$

where

$$
\begin{gathered}
\bar{\Gamma}_{0} \cup \bar{\Gamma}=\partial \Omega, \\
\Gamma_{0} \cap \Gamma=\varnothing, \\
\bar{\Gamma}_{0} \cup \overline{\Gamma^{\prime}}=\partial \Omega^{\prime}, \\
\Gamma_{0} \cap \Gamma^{\prime}=\varnothing .
\end{gathered}
$$

Then

$$
\frac{\partial v}{\partial n}(s)>\frac{\partial v^{\prime}}{\partial n}(s) \text { for all } s \in \Gamma_{0}
$$

Proof 3. Considering $v-v^{\prime}$, we have 


$$
\left\{\begin{array}{l}
\frac{\partial^{2}\left(v-v^{\prime}\right)}{\partial t^{2}}+p\left(v-v^{\prime}\right)-\Delta\left(v-v^{\prime}\right)=0 \text { in } \Omega^{\prime} \times(0, T), \\
v-v^{\prime}(x, t)=0 \text { on } \partial \Omega \times(0, T)=\Gamma_{0} \times(0, T), \\
v-v^{\prime}(x, 0)=v_{0}-v_{0}^{\prime}(x) \text { in } \Omega^{\prime}, \\
\frac{\partial}{\partial t}\left(v-v^{\prime}\right)(x, 0)=v_{1}-v_{1}^{\prime}(x) \text { in } \Omega^{\prime} .
\end{array}\right.
$$

By maximum principle (see [3]), $v \leq 0$ in $\Omega^{\prime}$.

Then, using again the maximum principle (see [3]), $(v-$ $\left.v^{\prime}\right)(x) \leq 0$ for all $x \in \Omega^{\prime}$. Let $x_{0} \in \Gamma_{0}$, and $x=x_{0}-n \tau \in \Omega$ where $n$ is exterior normal on $\Gamma_{0}$ and $\tau>0$. We suppose that $\left(v-v^{\prime}\right)(x)<\left(v-v^{\prime}\right)\left(x_{0}\right)=0$; in this case, by the Hopf lemma, we have

$$
\frac{\partial v}{\partial n}\left(x_{0}\right)>\frac{\partial v^{\prime}}{\partial n}\left(x_{0}\right)
$$

Lemma 5. If ( $\mathscr{H})$ is satisfied and $(\Omega, v)$ and $\left(\Omega^{\prime}, v^{\prime}\right)$ are two solutions of the free boundary problem

$$
\left\{\begin{array}{l}
\frac{\partial^{2} v}{\partial t^{2}}+p v-\Delta v=0 \text { in } \Omega \times(0, T), \\
v(x, t)=0 \text { on } \partial \Omega \times(0, T), \\
v(x, 0)=v_{0}(x) \text { in } \Omega, \\
\frac{\partial}{\partial t} v(x, 0)=v_{1}(x) \text { in } \Omega, \\
\frac{\partial v}{\partial n}(x, t)=k(\Omega) \text { on } \Gamma_{0} \times(0, T),
\end{array}\right.
$$

such that

$$
\begin{aligned}
& k(\Omega) \text { is the Lagrangian multiplier } \\
& \Omega^{\prime} \subset \Omega \\
& \left(\partial \Omega^{\prime} \cap \partial \Omega\right) \backslash \Gamma_{0} \neq \varnothing
\end{aligned}
$$

Then $k\left(\Omega^{\prime}\right)<k(\Omega)$.

Proof 4. We have

$$
\left\{\begin{array}{l}
\frac{\partial^{2}\left(v-v^{\prime}\right)}{\partial t^{2}}+p\left(v-v^{\prime}\right)-\Delta\left(v-v^{\prime}\right)=0 \text { in } \Omega^{\prime} \times(0, T), \\
v-v^{\prime}(x, t)=0 \text { on } \partial \Omega^{\prime} \cap \partial \Omega \times(0, T)=\Gamma_{0} \times(0, T), \\
v-v^{\prime}(x, 0)=v_{0}-v_{0}^{\prime}(x) \text { in } \Omega^{\prime}, \\
\frac{\partial}{\partial t}\left(v-v^{\prime}\right)(x, 0)=v_{1}-v_{1}^{\prime}(x) \text { in } \Omega^{\prime} .
\end{array}\right.
$$

Thanks to maximum principle (see [3]), $v \leq 0$ on $\Omega^{\prime}$ and $v-v^{\prime} \leq 0$ on $\Omega^{\prime}$. Let $x_{0} \in \partial \Omega^{\prime} \cap \partial \Omega$; then $\left(v-v^{\prime}\right)\left(x_{0}\right)=0$ and $x=x_{0}-n \tau \in \Omega^{\prime}$ where $n$ is exterior normal on $\Gamma_{0}$ and $\tau>0$. We suppose that $\left(v-v^{\prime}\right)(x)<\left(v-v^{\prime}\right)\left(x_{0}\right)=0$; in this case, by the Hopf lemma, we have

$$
\frac{\partial v}{\partial n}\left(x_{0}\right)>\frac{\partial v^{\prime}}{\partial n}\left(x_{0}\right) \text { this implies that } k(\Omega)>k\left(\Omega^{\prime}\right) .
$$

\section{Uniqueness and Convergence Result}

In this section, using results established in Section 4, we show the uniqueness of the domain $\Omega$ under some hypothesis, by following the methods of I. Ly et al. (see [15]). Most of the time, in the inverse problems, it is a great challenge to get uniqueness results. Now we are not able to produce a uniqueness result in the case where the unknown domain $\Omega$ is supposed to be star-shaped with respect to a fixed point $x_{0}, \in \Omega$. We think that it would be interesting to investigate this question. Our uniqueness result is obtained for any domains belonging to $B_{h}$, a class of geometrical sets in the Beurling sense of admissible domains satisfying an inequality constraint on the accessible boundary $\Gamma_{0}$ (see $[11,16])$. Another important hypothesis for our uniqueness result is the inclusion property in the following sense: one assumes that there are two domains in class $B_{h}$ and one of the two domains is included in the other. This is an interesting problem to weaken the inclusion property's hypothesis. The uniqueness result is started in Proposition 3.

Using results in Section 4, we show, under geometrical assumptions, that the domain $\Omega$ is unique. We have already assumed that one is able to estimate the normal derivative of $v_{\Omega}$ on $\Gamma_{0}$, i.e.,, there exists

$$
h: I R^{N} \longrightarrow I R_{-}^{*} \text { such that } \frac{\partial v_{\Omega}}{\partial n}=h \text { on } \Gamma_{0},
$$

where $n$ is the exterior unit normal vector field defined on $\Gamma_{0}$.

Let us define a class of geometrical sets in the Beurling sense (see, e.g., [15-17]).

Let $\Omega$ be a bounded domain which is uniformly Lipschitz and let $\Gamma_{0}$ be a subset of $I R^{N-1} \mathscr{C}^{2}$ that is regular. Let us take

$$
B_{h}=\left\{\Omega \subset I R^{N}, \Gamma_{0} \subset \partial \Omega, \frac{\partial v_{\Omega}}{\partial n} \leq h \text { on } \Gamma_{0}\right\},
$$

and $v_{\Omega}$ is solution to the following problem:

$$
\left\{\begin{array}{l}
\frac{\partial^{2} v}{\partial t^{2}}+p v-\Delta v=0 \text { in } \Omega \times(0, T), \\
v(x, t)=0 \text { on } \partial \Omega \times(0, T), \\
v(x, 0)=v_{0}(x) \text { in } \Omega \\
\frac{\partial}{\partial t} v(x, 0)=v_{1}(x) \text { in } \Omega .
\end{array}\right.
$$

By construction, $B_{h}$ is a nonempty set. In fact, this is because of Proposition 1 and measure assumption. We use 
set $B_{h}$ in the proof of Proposition 3. At first, we get the following lemma.

Lemma 6. If $(\mathscr{H})$ is satisfied, let $\Omega, \Omega^{\prime} \in B_{h}$; then $\Omega \cap \Omega^{\prime} \in B_{h}$.

Proof 5. Let $w$ be solution of

$$
\left\{\begin{array}{l}
\frac{\partial^{2} w}{\partial t^{2}}+p w-\Delta w=0 \text { in } \Omega \cap \Omega^{\prime} \times(0, T) \\
w(x, t)=0 \text { on } \partial \Omega \cap \Omega^{\prime} \times(0, T) \\
w(x, 0)=v_{0}(x) \text { in } \Omega \cap \Omega^{\prime} \\
\frac{\partial}{\partial t} w(x, 0)=v_{1}(x) \text { in } \Omega \cap \Omega^{\prime}
\end{array}\right.
$$

We have

$$
\left\{\begin{array}{l}
\frac{\partial^{2}\left(v-v^{\prime}\right)}{\partial t^{2}}+p\left(v-v^{\prime}\right)-\Delta\left(v-v^{\prime}\right)=0 \text { in } \Omega \cap \Omega^{\prime} \times(0, T) \\
v-v^{\prime}(x, t)=0 \text { on } \partial \Omega \times(0, T)=\Gamma_{0} \times(0, T) \\
v-v^{\prime}(x, 0)=v_{0}-v_{0}^{\prime}(x) \text { in } \Omega \cap \Omega^{\prime} \\
\frac{\partial}{\partial t}\left(v-v^{\prime}\right)(x, 0)=v_{1}-v_{1}^{\prime}(x) \text { in } \Omega \cap \Omega^{\prime}
\end{array}\right.
$$

By the maximum principle (see [3]), we show that

$$
v \leq 0 \text { and } v-w \leq 0
$$

Let $x_{0} \in \Gamma_{0}$, and we get

$$
\begin{aligned}
\frac{v\left(x_{0}-n \tau\right)-v\left(x_{0}\right)}{\tau} \leq & \frac{(v-w)\left(x_{0}-n \tau\right)-(v-w)\left(x_{0}\right)}{\tau} \text {, passing to the limit we get } \lim _{\tau \longrightarrow 0} \frac{v\left(x_{0}-n h\right)-v\left(x_{0}\right)}{\tau} \\
& \leq \lim _{\tau \longrightarrow 0} \frac{(v-w)\left(x_{0}-n \tau\right)-(v-w)\left(x_{0}\right)}{\tau}, \text { this implies that } \\
-\frac{\partial v}{\partial n} & \leq-\frac{\partial(v-w)}{\partial n} .
\end{aligned}
$$

Then,

$$
\frac{\partial(v-w)}{\partial n}\left(x_{0}\right) \leq \frac{\partial v}{\partial n}\left(x_{0}\right) \leq h \text { for all } x_{0} \in \Gamma_{0} .
$$

Then we get $\Omega \cap \Omega^{\prime} \in B_{h}$.

Proposition 3. Let $\Omega$ be a bounded domain and let $\Gamma_{0}, h, \Gamma$ be defined as in the Introduction. Consider the following Cauchy problem:

$$
\left\{\begin{array}{l}
\frac{\partial^{2} v}{\partial t^{2}}+p v-\Delta v=0 \text { in } \Omega \times(0, T) \\
v(x, t)=0 \text { on } \partial \Omega \times(0, T) \\
v(x, 0)=v_{0}(x) \text { in } \Omega \\
\frac{\partial}{\partial t} v(x, 0)=v_{1}(x) \text { in } \Omega \\
\frac{\partial v}{\partial n}(x, t)=h(x) \text { on } \Gamma_{0} \times(0, T)
\end{array}\right.
$$

Assume also that there are two domains $\Omega$ and $\Omega^{\prime}$, such that $\Omega^{\prime} \subset \Omega$ for which (49) is verified. Then, we have $\Omega=\Omega^{\prime}$.
Proof 6. Consider $\Omega, \Omega^{\prime}$ such that

$$
\left\{\begin{array}{l}
\frac{\partial^{2} v}{\partial t^{2}}+p v-\Delta v=0 \text { in } \Omega \times(0, T), \\
v(x, t)=0 \text { on } \partial \Omega \times(0, T)=\Gamma_{0} \times(0, T), \\
v(x, 0)=v_{0}(x) \text { in } \Omega, \\
\frac{\partial}{\partial t} v(x, 0)=v_{1}(x) \text { in } \Omega, \\
\frac{\partial^{2} v^{\prime}}{\partial t^{2}}+p v^{\prime}-\Delta v^{\prime}=0 \text { in } \Omega^{\prime} \times(0, T), \\
v^{\prime}(x, t)=0 \text { on } \partial \Omega^{\prime} \times(0, T)=\Gamma_{0} \times(0, T), \\
v^{\prime}(x, 0)=v_{0}^{\prime}(x) \text { in } \Omega^{\prime}, \\
\frac{\partial}{\partial t} v^{\prime}(x, 0)=v_{1}^{\prime}(x) \text { in } \Omega^{\prime} .
\end{array}\right.
$$

As $\Omega$ and $\Omega^{\prime} \in B_{h}$, we obtain $\Omega \cap \Omega^{\prime} \in B_{h}$.

Since $\Omega^{\prime} \subset \Omega$, we have $\Omega_{3}=\Omega \cap \Omega^{\prime}=\Omega^{\prime}$, and, by Lemma 6, we have $\partial v^{\prime} / \partial n \leq h$ on $\Gamma_{0}$.

Consider the following problem: 


$$
\left\{\begin{array}{l}
\frac{\partial^{2}\left(v-v^{\prime}\right)}{\partial t^{2}}+p\left(v-v^{\prime}\right)-\Delta\left(v-v^{\prime}\right)=0 \text { in } \Omega \cap \Omega^{\prime} \times(0, T) \\
v-v^{\prime}(x, t)=0 \text { on } \partial \Omega \times(0, T)=\Gamma_{0} \times(0, T) \\
v-v^{\prime}(x, 0)=v_{0}-v_{0}^{\prime}(x) \text { in } \Omega \cap \Omega^{\prime} \\
\frac{\partial}{\partial t}\left(v-v^{\prime}\right)(x, 0)=v_{1}-v_{1}^{\prime}(x) \text { in } \Omega \cap \Omega^{\prime}
\end{array}\right.
$$

We have (see [3])

$$
v \leq 0 \text { and } v-v^{\prime} \leq 0 .
$$

As $\Gamma_{0}$ satisfies the interior sphere condition, supposing that $v-v^{\prime}<0$, by the Hopf lemma, we get

$$
\frac{\partial v}{\partial n}>\frac{\partial v^{\prime}}{\partial n} \text { on } \Gamma_{0} \quad \text { i.e } h(x)>h(x) \forall x \in \Gamma_{0},
$$

which is false; then $\Omega=\Omega^{\prime}$.

\section{Stability of the Inverse Problem}

In this section, we establish the stability of solution $\Omega$ under some hypothesis too. The stability issue for this inverse problem seems important and difficult to be ignored. Recall the following lemma solving the Cauchy problem for the usual wave equation and giving energy and trace estimates of the solution.

Lemma 7. Let $\Omega$ be uniformly Lipschitzian included in $\mathbb{R}^{N}$, and a final time $T>0$.

$\left(v_{0}, v_{1}\right) \in H_{0}^{1}(\Omega) \times L^{2}(\Omega) \quad$ is given. Let $v \in C\left([0, T] ; H_{0}^{1}(\Omega)\right) \cap C^{1}\left([0, T] ; L^{2}(\Omega)\right)$ be the unique weak solution of the wave equation

$$
\left\{\begin{array}{l}
\frac{\partial^{2} v}{\partial t^{2}}+p v-\Delta v=0 \text { in } \Omega \times(0, T) \\
v(x, t)=0 \text { on } \partial \Omega \times(0, T)=\Gamma_{0} \times(0, T) \\
v(x, 0)=v_{0}(x) \text { in } \Omega \\
\frac{\partial}{\partial t} v(x, 0)=v_{1}(x) \text { in } \Omega .
\end{array}\right.
$$

Then there exists a constant $C(\Omega, T)>0$ (which depends only on $\Omega$ and $T$ ) such that, for all $t \in[0, T]$,

$$
\begin{aligned}
& \int_{\Omega}\left|\frac{\partial v}{\partial t}(x, t)\right|^{2} \mathrm{~d} x+\int_{\Omega}|\nabla v(x, t)|^{2} \mathrm{~d} x \\
& \quad \leq C\left(\int_{\Omega} v_{1}(x)^{2} \mathrm{~d} x+\int_{\Omega}\left|\nabla v_{0}(x)\right|^{2} \mathrm{~d} x\right) .
\end{aligned}
$$

The normal derivative $\partial v / \partial n$ belongs to $L^{2}\left([0, T] ; L^{2}(\partial \Omega)\right)$ and verifies

$$
\left\|\frac{\partial v}{\partial n}\right\|_{\left.L^{2}([0, T]] ; L^{2}(\partial \Omega)\right)} \leq C\left(\left\|v_{0}\right\|_{H_{0}^{1}(\Omega)}+\left\|v_{1}\right\|_{H_{0}^{1}(\Omega)}\right) .
$$

This result is very classical; estimate (20) can be formally deduced from the multiplication of (19) by $\partial v / \partial t$ and then by part integrations of this equality on $[0, T] \times \Omega$. Concerning estimate (56), we refer to [18]. This is a hidden regularity result that can be proved using the method of multipliers.

Proposition 4. Let $\Omega$ be a bounded uniformly Lipschitz domain and let $\Gamma_{0}, \Gamma_{0}^{\prime}$ be subsets of $I R^{N-1} \mathscr{C}^{2}$ that is regular such that

$$
\begin{aligned}
& \bar{\Gamma}_{0} \cup \bar{\Gamma}=\partial \Omega, \\
& \Gamma_{0} \cap \Gamma=\varnothing, \\
& \overline{\Gamma_{0}^{\prime}} \cup \overline{\Gamma^{\prime}}=\partial \Omega, \\
& \Gamma_{0}^{\prime} \cap \Gamma^{\prime}=\varnothing .
\end{aligned}
$$

Assume that (49) is verified. Then

there is $\eta>0$ such that $d_{H}\left(\partial \Omega \backslash \Gamma, \partial \Omega \backslash \Gamma^{\prime}\right)$

$$
<\eta\left\|\left.\frac{\partial v}{\partial n}\right|_{\Gamma^{\prime}}-\left.\frac{\partial v}{\partial n}\right|_{\Gamma}\right\|_{L^{2}\left([0, T] ; L^{2}(\partial \Omega)\right)} .
$$

This inequality (58) describes the Lipschitz stability of the inverse problem.

Proof. From (56), $\|\partial v / \partial n\|_{L^{2}\left([0, T]: L^{2}(\partial \Omega)\right)}$ is bounded; then there exists $\eta>0$ such that

$\|\partial v / \partial n\|_{L^{2}\left([0, T]: L^{2}(\partial \Omega)\right)} \geq \eta$. Then we have

$$
\begin{aligned}
& \left\|\left.\frac{\partial v}{\partial n}\right|_{\Gamma^{\prime}}-\left.\frac{\partial v}{\partial n}\right|_{\Gamma}\right\| L_{L^{2}\left([0, T] ; L^{2}(\partial \Omega)\right)} \geq\left|\left\|\chi_{\Gamma^{\prime}} \frac{\partial v}{\partial n}\right\|_{L^{2}\left([0, T] ; L^{2}(\partial \Omega)\right)}-\left\|\chi_{\Gamma} \frac{\partial v}{\partial n}\right\| \|_{L^{2}\left([0, T] ; L^{2}(\partial \Omega)\right)}\right| \\
& \geq \eta\left|\Gamma^{\prime} \Delta \Gamma\right| \geq \eta d_{H}\left(\partial \Omega \backslash \Gamma^{\prime}, \partial \Omega \backslash \Gamma\right) .
\end{aligned}
$$




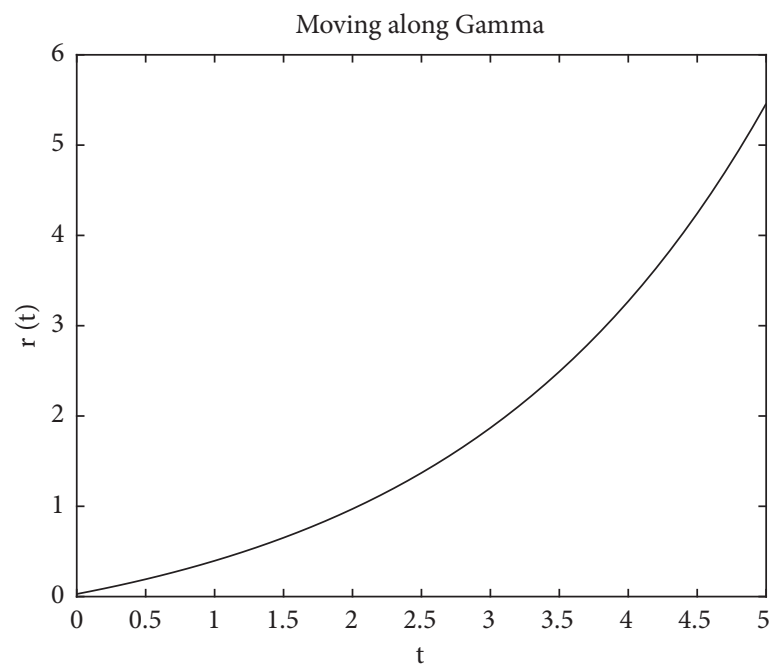

Figure 1: $T=5, N=300$, and theta $=\mathrm{pi} / 28$.

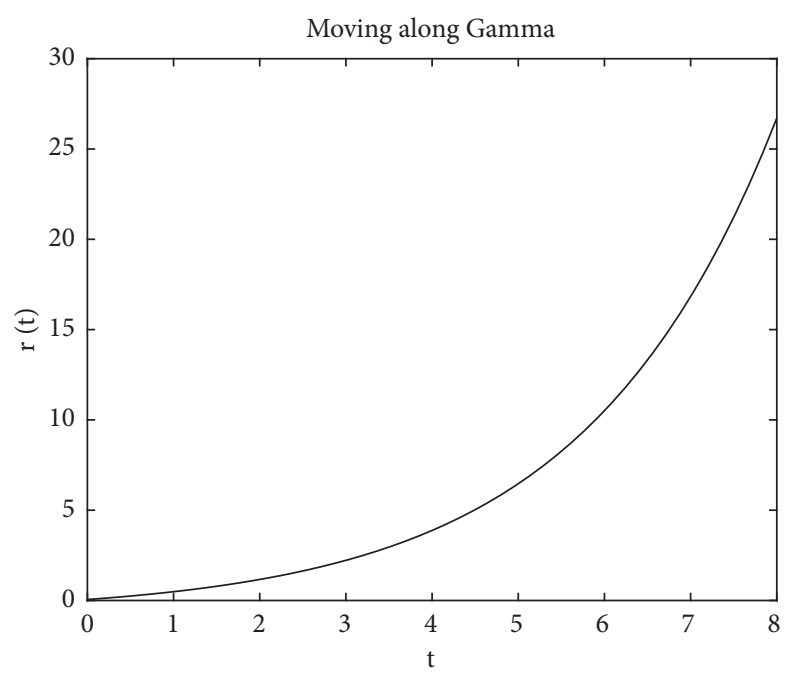

Figure 2: $T=8, N=300$, and theta $=\mathrm{pi} / 18$.

\section{Numerical Simulations}

In this part, we solve our inverse problem numerically using polar coordinates. Let $h$ be a function as defined in the
Introduction; we seek to determine $\Gamma$ of class $\mathscr{C}^{2}$ under the constraints given. We choose function $h$ defined by

$$
h(r, \theta)=\sum_{n=0}^{N} e^{-\sqrt{n}+\sqrt{\lambda_{n}} t}(\sin (\theta) \cos (n r \sin (\theta)) \operatorname{ch}(n r \cos (\theta))+\cos (\theta) \sin (n r \sin (\theta)) \operatorname{sh}(n r \cos (\theta))),
$$

with $N \in \mathbb{N}, \lambda_{n} \in \mathbb{R}_{+}$, ch the hyperbolic cosine function and $s h$ the hyperbolic sine function. The integer $n$ takes values from 0 to $N$ and $\lambda_{n}$ is a multiple for $r$ depending on $n$. We consider that $h$ is the normal derivative of a function $u$ unique solution of problem 1 in a domain $\Omega$ of which a part $\Gamma$ of the boundary is unknown. We seek to determine its optimal shape of $\Gamma$. With Matlab, by varying the parameters $N, \lambda_{n}, \theta$ and $t$, we determine $\Gamma$ of class $\mathscr{C}^{2}$ which we describe as a trajectory. Thus, we choose arbitrary values for $r$ and $\lambda_{n}$ and a maximum value for time $t$. By varying the angle $\theta$ and $N$, we obtain the geometrical optimal shapes of $\Gamma$ in the below figures. For each given unit of time, 


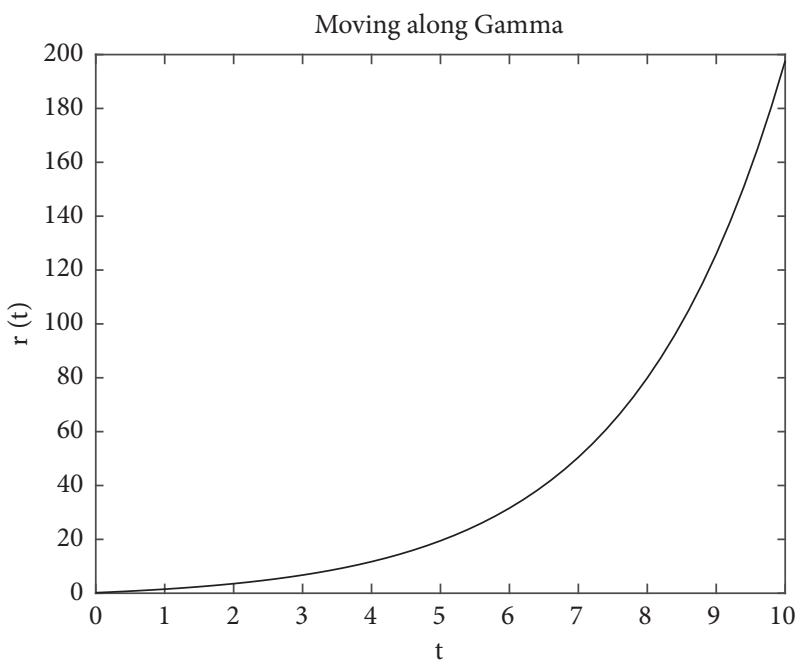

Figure 3: $T=10, N=300$, and theta $=\mathrm{pi} / 30$.

we vary $N$ and $\theta$. Thus, for each fixed time, we obtain an optimal shape when $N=300$ and $\theta$ is equal to the value indicated in the legend.

In Figure 1 where the observation time is 5 seconds for a maximum angle equal to $300 \times \pi / 28$, the length of $\Gamma$ is equal to 5.43 kilometers.

In Figure 2 where the observation time is 8 seconds for a maximum angle equal to $300 \times \pi / 18$, the length of $\Gamma$ is equal to 23.79 kilometers.

In Figure 3 where the observation time is 10 seconds for a maximum angle equal to $10 \pi$, the length of $\Gamma$ is equal to 195.15 kilometers.

We notice that if the observation time is small, then the optimal shape of $\Gamma$ looks like an arc of circle. But if it is larger, the optimal shape of $\Gamma$ is a part of a hyperbola.

\section{Conclusion}

In this paper, we prove the existence result of the solution for our inverse problem by determining the optimal shape in Section 2 and we prove the existence of a Lagrange multiplier, which appears in the optimality condition of the problem. By maximum principle for hyperbolic equations, we prove the uniqueness and the Lipschitz stability of the solution for our inverse problem. We make some numerical simulations with Matlab to illustrate the theoretical results and then identify the optimal shape of $\Gamma$. It would be interesting to study the problem with nonsmooth boundaries; topological optimization is the technique to use in future researches.

\section{Data Availability}

The data used to support the findings of this study are included within the article.

\section{Conflicts of Interest}

The authors declare that there are no conflicts of interest.

\section{References}

[1] R. Gilbarg and N. S. Trudinger, Elliptic Partial Differential Equations of Second Order, Springer-Verlag, Berlin, Germany, second edition, 1983.

[2] J.-L. Lions and E. Magenes, Non Homogenenous Boundary Value Problem and Applications, Springer-Verlag, Berlin, Germany, 1972.

[3] M. H. Protter and H. F. Weinberger, Maximum Principles in Differential Equations, Springer-Verlag New York. Inc., Berlin, Germany, 1984.

[4] M. V. Klibanov, "Inverse problems and Carleman estimates," Inverse Problems, vol. 8, no. 4, pp. 575-596, 1992.

[5] M. Yamamoto, "Uniqueness and stability in multidimensional hyperbolic inverse problems," Journal de Mathematiques Pures et Appliquees, vol. 9, no. 1, pp. 78-98, 1999.

[6] L. Baudouin, Inverse Problems and Robust Control of Some Partial Differential Equations, Universit Paul Sabatier-Toulouse III, Toulouse, France, 2014, http://hal.archives-ouvertes. $\mathrm{fr} / \mathrm{hal}-01067485 / \mathrm{fr} /$.

[7] L. Baudouin and J.-P. Puel, "Uniqueness and stability in an inverse problem for the Schr dinger equation," Inverse Problems, vol. 18, no. 6, pp. 1537-1554, 2002.

[8] V. Isakov and A. Friedman, Inverse Problems for Partial Differential Equations, Springer Science + Business Media, Inc., vol. 127, pp. 20-205, Berlin, Germany, second edition, 2006.

[9] M. Kac, "Can one hear the shape of a drum?" The American Mathematical Monthly, vol. 73, no. 4, pp. 1-23, 1966.

[10] R. Kress, "Inverse problems and conformal mapping," Mathematics and Computers in Simulation, vol. 66, Article ID 123013, 2004.

[11] A. Henrot, M. Pierre, Variation and Shape Optimization:A Geometric Analysis, Springer-Verlag, Berlin, Germany, 2005.

[12] E. DiBenedetto, " $\mathrm{C}^{1+\alpha}$ local regularity of weak solutions of degenerate elliptic equations," Nonlinear Analysis: Theory, Methods \& Applications, vol. 7, no. 8, pp. 827-850, 1983.

[13] P. Tolksdorf, "Regularity for a more general class of quasilinear elliptic equations," Journal of Differential Equations, vol. 51, no. 1, pp. 126-150, 1984.

[14] J. Sokolowsi and J. Zolesio, "Introduction to shape optimization: shape sensitivity," Springer Series in Computational Mathematics, vol. 16, 1991. 
[15] L. Ndiaye, I. Ly, and D. Seck, "A shape reconstruction problem with the Laplace operator," Bulletin of Mathematical Analysis and Applications, vol. 4, no. 1, pp. 91-103, 2012, ISSN: 18211291.

[16] D. Seck, Etude d'un problme frontire libre de type Bernoulli, p. 505, Thse d' Universit de Franche- Comt, Besançon, France, 1996.

[17] A. Henrot, "Subsolutions and Supersolutions in a free boundary problem," Arkiv för Matematik, vol. 32, no. 1, pp. 79-98, 1994.

[18] J.-L. Lions, "Exact Controllability, stabilisation and disturbances of distributed systems, tome 1," Exact Controllability, vol. 8, 1988. 